\title{
Improved design of low-pressure fluidic microvalves
}

\author{
Anna Pandolfi ${ }^{1}$ and Michael Ortiz ${ }^{2}$ \\ ${ }^{1}$ Dipartimento di Ingegneria Strutturale, Politecnico di Milano, Piazza Leonardo da Vinci 32, \\ 20133 Milano Italy \\ ${ }^{2}$ Engineering and Applied Sciences Division, California Institute of Technology, \\ 1200 E California Blvd, Pasadena, CA 91125 USA \\ E-mail: pandolfi@stru.polimi.it and ortiz@aero.caltech.edu
}

Received 20 March 2007, in final form 5 June 2007

Published 29 June 2007

Online at stacks.iop.org/JMM/17/1487

\begin{abstract}
Multilayer soft lithography (MSL) is used to fabricate monolithic elastomeric on-off microvalves by adopting a two-layer cross-channel architecture. The performance of microvalves is strongly dependent on the two-channel geometry (width, height and shape) and on the thickness of the interlayer membrane. Using a finite element model previously validated against experiments, we propose a new fluidic microvalve design, based on the concept of chemically swelling the thin interlayer membrane so as to induce two stable equilibrium configurations. The complete closure of the new valve may then be achieved by applying a much reduced actuation pressure, down to $1 / 4$ of the pressure needed by the standard monostable design. The maximum stress in the interlayer membrane of the bistable valve also drops to $1 / 3$ of the value corresponding to the standard design.
\end{abstract}

(Some figures in this article are in colour only in the electronic version)

\section{Introduction}

Soft lithography (SL) is an alternative technique to siliconbased micromachining and conventional photolithography for fabricating fluidic structures at microscale and mechanical structures at nanoscale. Soft lithography originates in the late 1980s from the work of Whitesides and collaborators [1]. SL uses an elastomeric stamp (mold) to pattern a wide variety of materials, such as organic polymers, colloids, inorganic solids and proteins. Many advantages are connected to its use. Elastomers are tolerated by a wide range of materials commonly used in chemistry, biochemistry and biology, and are compatible with many substrates (glass, plastic, ceramics). SL devices are easy to fabricate; elastomers are very soft materials - thus the technique allows for rapid prototyping and low cost.

SL requires a master stamp, which can be obtained by micromachining or photolithography, with a negative pattern. The fluid poly(dimethylsiloxane) (PDMS) is poured over the master stamp and cured, resulting into a positive stamp (or mold) with patterned relief structures on its surface. At the end of the process, the master stamp is removed (see figure 1).
Unger et al [2] improved SL in order to obtain multilayer devices. They use a two-component, addition-cure silicone rubber and bond several layers together, varying the relative composition of the silicone rubber between two adjacent layers. In practice, two bonding layers with different composition are cast separately against microfabricated master stamps. After curing, the second stamp is placed on top of the first layer, forming a hermetic seal (made irreversible by a further curing), which possesses the same resistance as the bulk material. The assembled device is then sealed against a glass substrate (figure 2). This modified technique is called multilayer soft lithography (MSL), and it overcomes many issues of the original SL. In fact, MSL avoids interlayer adhesion failures and thermal stress problems, allows for large deflections with small actuation forces, and permits the reduction of the device dead volume up to $1 \mathrm{pl}$, i.e., more than two orders of magnitude compared with silicon-based devices. As a main result, the maximum on-off frequency of such microdevices reaches 70-100 Hz. MSL allows us to combine microvalves to produce peristaltic micropumps. More complex applications include the fabrication of microfluidic chip platforms [3]. 


\section{Obtain Master by Photolitography

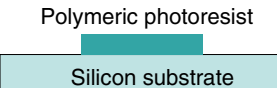

Pour PDMS over Master
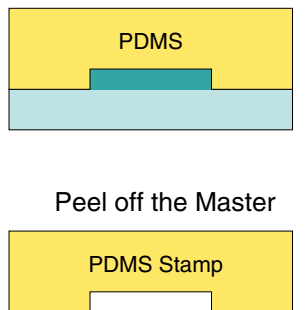

Figure 1. Soft lithography. Fluid PDMS is poured on a silicon substrate (polymeric photoresist). After curing, it is peeled off from the master.

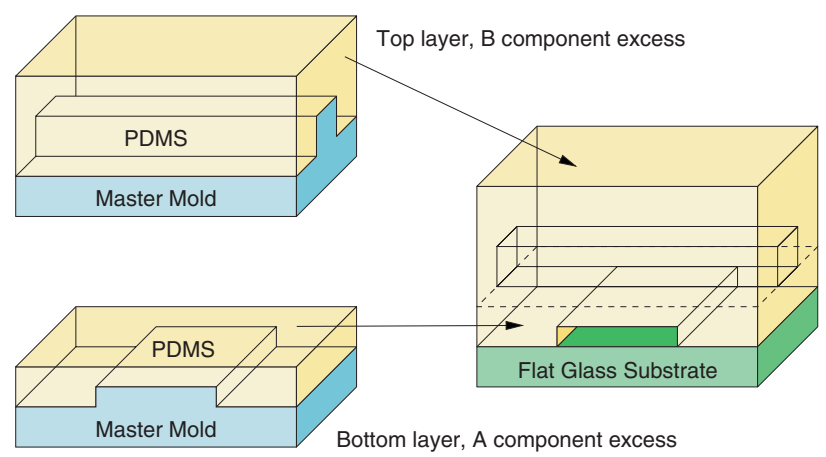

Figure 2. Multilayer soft lithography assembly. Two bonding layers, with different composition, are cast separately. After curing, the second layer is placed on top of the first. The resulting device is further cured.

A cross-channel architecture is used to fabricate monolithic microvalves (figure 3). Two orthogonal channels are separated by a thin $(3-10 \mu \mathrm{m})$ membrane. One of the channels acts as control (actuation channel). The second channel is used for the fluid flow (fluidic channel). When pressure is applied to the actuation channel, the membrane deflects and eventually closes the fluidic channel. The typical response time of such devices is $1-2 \mathrm{~ms}$, and the actuation pressure range is $5-50 \mathrm{kPa}$. The performance of a microvalve
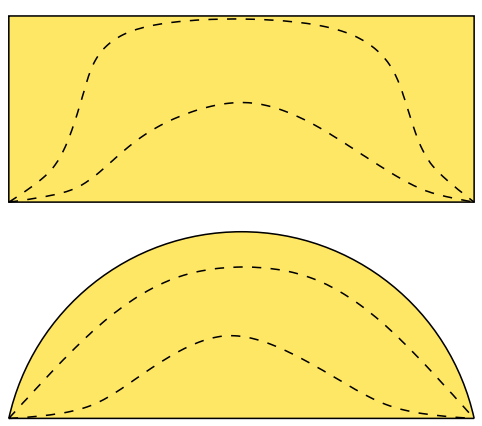

Figure 4. Influence of the channel shape on the microvalve closure.

is strongly dependent on the geometry (width, height and shape) of the fluidic channel; for example, a rectangular or trapezoidal section does not allow complete closure of the microvalve, whereas round channels work better (figure 4). A circular shape of the fluidic channel is thus adopted in the fabrication of microdevices.

An appealing feature of miniaturized valves is the low actuation pressure, which allows for a high device frequency. MSL valves with a circular section of the fluidic channel need actuation pressures in the range $5-30 \mathrm{kPa}$. In a combined experimental and numerical investigation, Studer et al [4] evaluated the dependence of actuation pressure on geometrical parameters of the microvalve. In particular, the dependence on membrane thickness and actuation channel width was analyzed. The actuation pressure increases linearly with membrane thickness and decreases exponentially with channel width. At complete valve closure, the normal strain in the membrane reaches $8 \%$. The corresponding maximum normal stress is about 0.2-0.3 MPa.

In this paper, we show that closure can be assisted by out-of-plane buckling of the membrane caused by controlled chemical swelling. Thus, suppose that a swelling solvent is applied to the membrane of a microvalve. Upon swelling the membrane wants to stretch within its plane but is constrained by the valve, resulting in a state of residual compression. If the swelling volumetric strain is large enough, the membrane spontaneously buckles out-of-plane in order to reduce its energy. Two stable buckled configurations exist on either side of the membrane, one in the fluidic channel and another in the actuation channel. The post-buckling deflection of the membrane may be sufficient to close the fluidic channel or

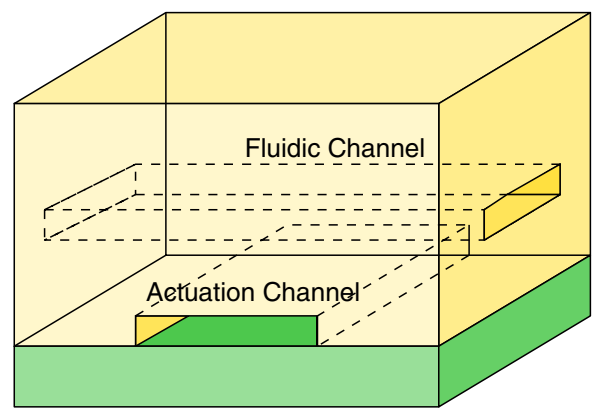

(a)

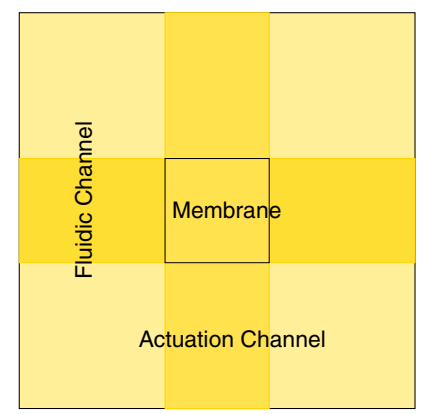

(b)

Figure 3. Monolithic microvalve. Two channels are assembled into a cross shape, defining a thin membrane which acts as an on-off valve. 
may only partially close the channel. In either case, we show by means of detailed finite-element calculations that the pressure required for the reversible actuation of the valve is much reduced below the actuation pressure of the untreated membrane.

As suggested by the brief review presented in section 2, the requisite chemical swelling may be induced by injecting silicone oil into Poly(dimethylsiloxane) PDMS actuation channels. Thus, once the cross-channel architecture of a microvalve has been built, silicone oil can be circulated within the actuation channel long enough to saturate the microvalve membranes. The solvent reacts with the elastomer of the channel walls (up to a certain depth) and of the membrane. Our calculations show that a moderate (15-20\%) volume increase (corresponding to 1.04-1.06 stretch) is sufficient to buckle the membrane, without affecting the functionality of the channel.

\section{Chemical swelling}

Poly(dimethylsiloxane) (PDMS), commonly referred as silicone rubber, is the material of choice for many microfluidic device applications [1, 2, 5, 6]. PDMS offers numerous attractive properties in relation to microfluidics. Upon crosslinking, it becomes an elastomeric material with a low Young's modulus $(\approx 750 \mathrm{kPa}$, in contrast to the much higher value of 100-150 GPa for silicon) capable of conforming to mold surfaces and forming reversible seals. It also has a low surface energy $\left(\approx 20 \mathrm{erg} \mathrm{cm}^{-2}=0.02 \mathrm{~N} \mathrm{~m}^{-1}\right)$, which facilitates easy release from molds after patterning. Furthermore, PDMS exhibits a high gas permeability, which allows gas bubbles within channels to permeate out of the device and helps to sustain cells and micro-organisms inside the device. The PDMS non-toxic nature also renders it well suited to medical applications.

Another notable characteristic of PDMS is that it swells in most organic solvents [7]. In particular, hexanes, ethyl ether, toluene, dichloromethane, acetone and acetonitrile greatly swell the material. The solvent compatibility of PDMS-based microfluidic devices has been recently investigated [7]. Lee et al provided swelling data for a large set of PDMS solvents. According to [7], the solubility of a polymer is related to its cohesive energy density $c$, i.e., the energy associated with the intermolecular attractive interactions within a unit volume of material. For two materials to be soluble, their cohesive energy must be similar, since this energy must be overcome in order to separate the solute molecules and insert the solvent molecules. The solubility parameter, $\delta=\sqrt{c}$, thus provides a useful indicator for predicting the swelling behavior of a polymer in the absence of more precise information. Lee et al showed that the relationship between $\delta$ and swelling (polymer modified weight/polymer dry weight ratio) is not linear and differs for each polymer-solvent system. It should also be carefully noted that swelling is not the only consequence of polymer mixing. Additional modifications of the elastomer properties are also observed, including: changes in the surface energy of the elastomers; swelling of elastomers bonded on glass may induce residual stresses; some solvents react with PDMS and break the polymer into smaller subunits; PDMS treated with high-solubility solvents can de-swell back to its original shape after the removal of the solvent; and highly swelling solvents evaporate quickly when exposed to air.

The stability in time of the polymer-solvent mixing is crucial in order to exploit the swelling properties of PDMS in microvalve design. Generally, a polymer-solvent mixing is permanent in time if solvent and polymer possess a similar chemical nature. Silicon oils have a chemical structure close to that of PDMS, which make them good candidates for stable mixing. Experimental data concerning the behavior of elastomer and silicone oil are available in the literature. Mathison et al [9] analyzed the behavior of a two-component elastomer (modified RTV compound, known as Aircast 3700) and a silicone oil (type $47 \mathrm{~V} \mathrm{100)}$ at different temperatures $\left(0-150{ }^{\circ} \mathrm{C}\right)$. Silicon oils show a smaller absorption than hydrocarbon solvents. The maximum weight gain is limited to $16-20 \%$ of the polymer dry weight, whereas hydrocarbons can swell a polymer up to $200 \%$ of the original weight. Unlike hydrocarbons, the swelling of PDMS by silicone oils is permanent and irreversible. The relationship between elastomer volume change and fluid absorbed is linear at all temperatures, with a small temperature dependence both in absorption and swelling (decreasing with temperature increase) [9]. Because of these properties, silicon oil suggests itself as the solvent of choice for inducing localized swelling of PDMS in the design of bistable microvalves.

\section{Finite element simulations}

We proceed to quantify the effect of chemical swelling on the performance of microvalves by way of detailed finite-element calculations. The numerical model of the microvalve accounts for large displacements, large strains, material nonlinearity and non-smooth contact. The stable equilibrium configurations of the microvalve are computed by dynamic relaxation [10]. In calculations we specifically adopt the implementation proposed by Oakley and Knight [11]. Frictionless contact between membrane and channel ceiling is enforced by means of a non-smooth contact algorithm [12-14]. The fidelity of the numerical model has been carefully validated against experimental data by Studer et al [4].

\subsection{Material model}

The total deformation gradient $\boldsymbol{F}$ can be decomposed as

$$
\boldsymbol{F}=\boldsymbol{F}^{e} \boldsymbol{F}^{*}
$$

into an elastic deformation $\boldsymbol{F}^{e}$ and an eigendeformation $\boldsymbol{F}^{*}$. Here and subsequently, a boldface symbol denotes the matrix of components of a $3 \times 3$ tensor. The eigendeformation characterizes the new stress-free or natural configuration induced by chemical swelling, whereas $\boldsymbol{F}^{e}$ is an elastic residual state of deformation caused by the incompatibility of $\boldsymbol{F}^{*}$. The eigendeformation is volumetric and therefore of the form

$$
\boldsymbol{F}^{*}=\lambda^{*} \boldsymbol{I}
$$

where $\lambda^{*}$ is an eigenstretch and $\boldsymbol{I}$ is the identity tensor. The volume ratio after and prior to swelling is given by the Jacobian

$$
J^{*}=\operatorname{det} \boldsymbol{F}^{*}=\lambda^{* 3} \text {. }
$$




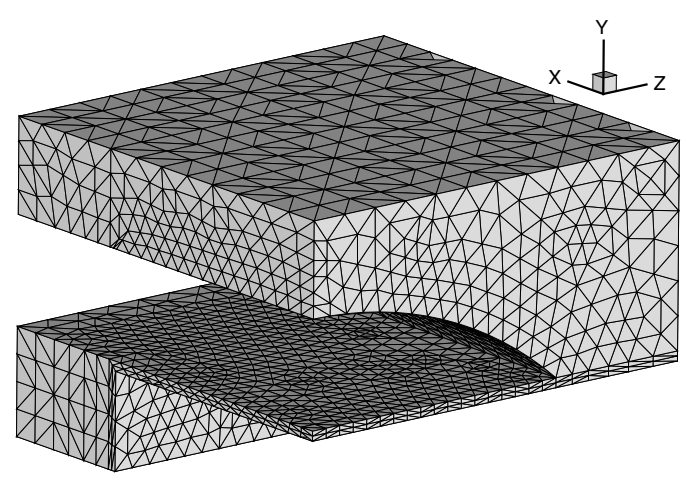

Figure 5. Mesh used in the calculations, consisting of 3898 nodes and 2021 ten-node tetrahedral elements.

The elasticity of the material is described by means of a neoHookean model (cf, e.g., [15]) extended to the compressible range. The assumed elastic strain-energy density of the material per unit undeformed volume is

$$
W^{e}\left(\boldsymbol{F}^{e}\right)=\frac{1}{2} \lambda \log ^{2} J^{e}+\frac{1}{2} \mu\left(\left|\boldsymbol{F}^{e}\right|^{2}-3-2 \log J^{e}\right),
$$

where $\mu$ and $\lambda$ are the Lamè constants and $J^{e}=\operatorname{det}\left(\boldsymbol{F}^{e}\right)$ is the elastic volume deformation. We recall that the components of the first Piola-Kirchhoff stress tensor $\boldsymbol{P}$ coincide with the tractions per unit undeformed area acting on coordinate planes in the undeformed configuration. The first Piola-Kirchhoff stress tensor follows from the strain energy (4) as

$$
\boldsymbol{P}=\frac{\partial W^{e}}{\partial \boldsymbol{F}}=\frac{\partial W^{e}}{\partial \boldsymbol{F}^{e}} \frac{\partial \boldsymbol{F}^{e}}{\partial \boldsymbol{F}}=\lambda^{*-1} \boldsymbol{P}^{e}
$$

where

$$
\boldsymbol{P}^{e} \equiv \frac{\partial W^{e}}{\partial \boldsymbol{F}^{e}}=\left(\lambda \log J^{e}-\mu\right) \boldsymbol{F}^{e-T}+\mu \boldsymbol{F}^{e} .
$$

We also recall that the components of the Cauchy stress tensor $\sigma$ coincide with the tractions per unit deformed area acting on coordinate planes in the deformed configuration. The Cauchy stress follows as

$$
\boldsymbol{\sigma}=J^{-1} \boldsymbol{P} \boldsymbol{F}^{T}=J^{*-1} J^{e-1} \boldsymbol{P}^{e} \boldsymbol{F}^{e T}=J^{*-1} \boldsymbol{\sigma}^{e}
$$

where $J=\operatorname{det} \boldsymbol{F}$ is the total volume change,

$$
\boldsymbol{\sigma}^{e} \equiv J^{e-1} \boldsymbol{P}^{e} \boldsymbol{F}^{e T}=\left(\lambda \log J^{e}-\mu\right) \boldsymbol{I}+\mu \boldsymbol{B}^{e},
$$

and $\boldsymbol{B}^{e}=\boldsymbol{F}^{e} \boldsymbol{F}^{e T}$ is the elastic left Cauchy-Green deformation tensor. We also note for subsequent reference that the Mises stress is computed from the components of the Cauchy stress as

$$
\sigma_{M}=\sqrt{\sigma_{1}^{2}+\sigma_{2}^{2}+\sigma_{3}^{2}-\sigma_{12}^{2}-\sigma_{23}^{2}-\sigma_{31}^{2}} .
$$

\subsection{Finite element model}

A standard geometry of a fluidic microvalve is discretized into ten-node quadratic tetrahedral finite elements with four integration points. Owing to the two-fold symmetry of the problem, the computational model is restricted to one-fourth of the microvalve. The geometry of the valve and its finite element discretization is shown in figure 5. The size of the discretized volume is $225 \times 225 \times 160 \mu \mathrm{m}$. The actuation channel is rectangular $(300 \times 50 \mu \mathrm{m})$ and the fluidic channel
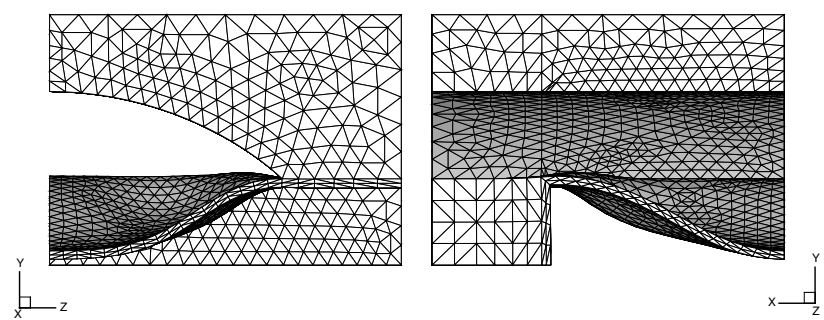

$\downarrow_{x}$
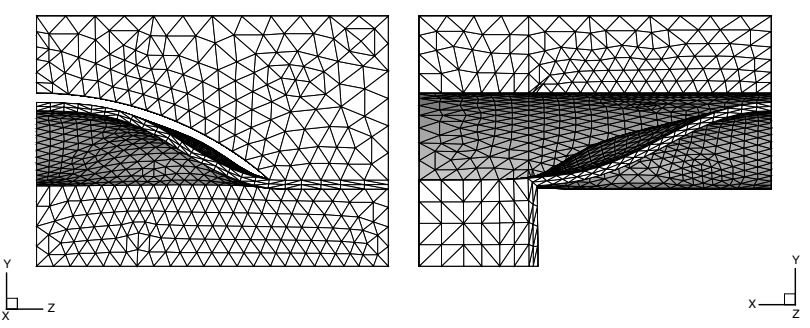

Figure 6. The two buckled configurations of the bistable microvalve. Top: open configuration, lateral views. Bottom: closed configuration, lateral views.

is round $(300 \times 56 \mu \mathrm{m})$. The thickness of the membrane is $5 \mu \mathrm{m}$. The number of nodes is 3898 , and the number of elements is 2021.

Numerical calculations are performed in two stages. The first stage concerns the PDMS swelling induced by the solvent flow in the actuation channel. The swelling is simulated by applying a volumetric change to the elements surrounding the actuation channel through a depth equal to the membrane thickness. Owing to the slenderness of the membrane, a moderate $(5-7 \%)$ volume change causes the membrane to buckle, partially closing one of the two channels. In the second stage, a uniform pressure is applied within the actuation channel and increased until complete closure of the fluidic channel is achieved.

Figure 6 shows the predicted buckled configurations of the swollen membrane. A 5\% volumetric deformation is initially applied to channel walls and valve membrane and the fluidic channel is not pressurized. The open configuration is reached spontaneously at the end of the swelling stage, with zero pressure in both channels. In order to obtain the closed configuration a pressure cycle $0 \rightarrow 2 \rightarrow 0 \mathrm{kPa}$ is applied in the actuation channel. We note that both buckled configurations are stable under zero pressure. It should also be noted that the zero-pressure closed configuration does not fully close the fluidic channel. To achieve closure, an additional pressure must be applied in the actuation channel. The key design question therefore concerns the level of pressure required for closure in the bistable and, specifically, how that pressure relates to that of a standard monostable valve configuration.

\subsection{Results}

Comparative results of numerical calculations for monostable and bistable valves are shown in figures 7-8. The thickness of the membrane adopted in the calculations is $5 \mu \mathrm{m}$. In this case, the actuation pressure required for the complete closure of an empty fluidic channel is $8 \mathrm{kPa}$ for the monostable valve, figure $7(a)$, and $2 \mathrm{kPa}$ for the bistable valve, figure $7(b)$, or 


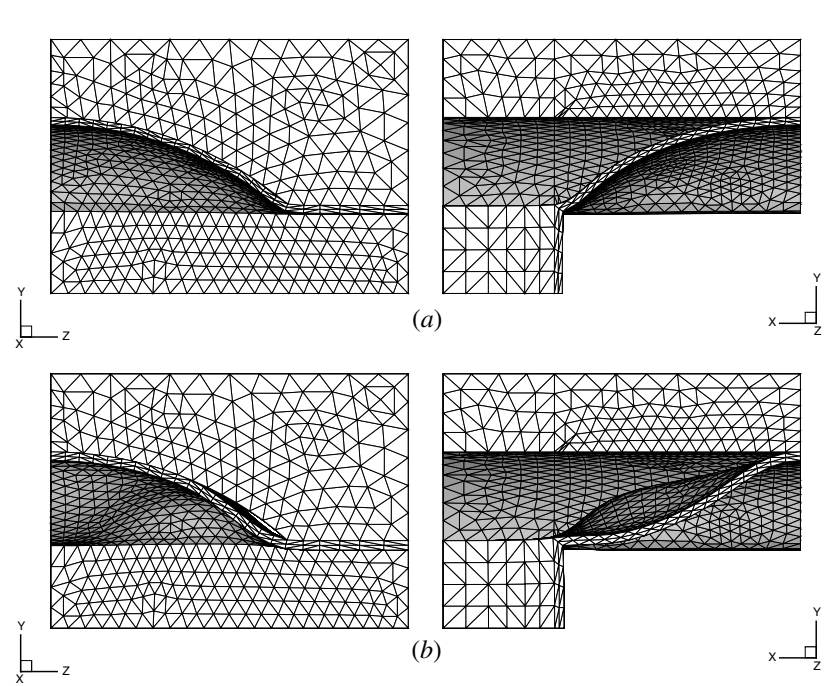

Figure 7. Configuration of the membrane at valve closed: (a) standard microvalve, $8 \mathrm{kPa}$ actuation pressure, lateral views; (b) bistable microvalve, $2 \mathrm{kPa}$ actuation pressure, lateral views.

a reduction of $75 \%$. Figure 8 compares the Mises equivalent stresses the monostable and bistable valves at closure. The maximum Mises stress is $0.24 \mathrm{MPa}$ for the standard valve and drops to $0.07 \mathrm{MPa}$ for the bistable valve.

In order to gain additional insight into the relative performance of the monostable and bistable valves, figure $9(a)$ compiles the actuation pressures for membrane thicknesses in the range $5-15 \mu \mathrm{m}$ for at constant $5 \%$ volume increase. We begin by considering the case in which the fluidic channel is empty. The figure also includes experimental data for the standard monostable valve [4] by way of validation of the numerical approach. The actuation pressure for the bistable design is consistently of the order of $1 / 4$ the activation pressure for the standard monostable valve. This gain in performance confers the bistable valve a substantial design advantage. The reduction in actuation pressure is accompanied by a simultaneous reduction, of the order of $1 / 3$, of the operating level of stress, figure $9(b)$, which may be expected to improve the durability of the valve.

Figure 10 shows the effect of fluidic pressure in the range of 0 to $10 \mathrm{kPa}$ on the performance of the valve for the $5 \mu \mathrm{m}$ membrane thickness. Other than an offset of the actuation pressure equal to the fluidic pressure, the closure response of the valve remains essentially identical to that of the empty fluidic channel. In particular, the level of stress remains ostensibly unchanged. By way of contrast, when the fluidic pressure is larger than $0.4 \mathrm{kPa}$, the membrane relaxes to the open buckled configuration upon release of the actuation pressure, which differs from the release behavior at fluidic pressures less than $0.4 \mathrm{kPa}$ in which the membrane relaxes to the closed buckled configuration.

The performance of the microvalve against leakage has been evaluated by measuring the valve opening area at the cross section for different values of actuation and fluidic pressures. The area is normalized conveniently over the nominal cross area of the standard valve. In our calculations, the actuation pressure is applied in small steps of $0.4 \mathrm{kPa}$, enabling the evaluation of the valve opening as a function of both actuation pressure and fluidic pressure. This dependence is shown in figure 11 , where the normalized valve opening is reported as a function of the pressure differential, i.e., difference between actuation and fluidic pressure, and of the fluidic pressure. The plot shows that the pressure differential that actuates the valve, i.e. $2 \mathrm{kPa}$ for the $5 \mu \mathrm{m}$ membrane thickness, is not affected

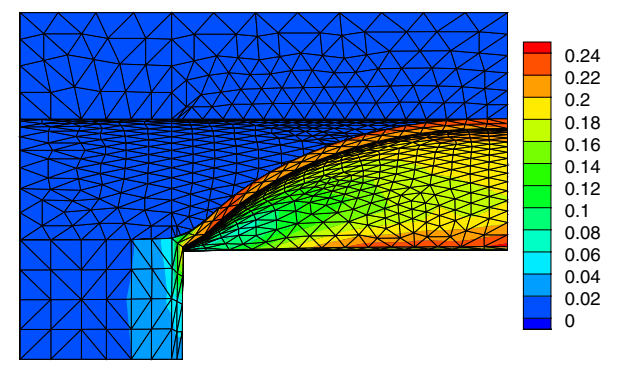

(a)
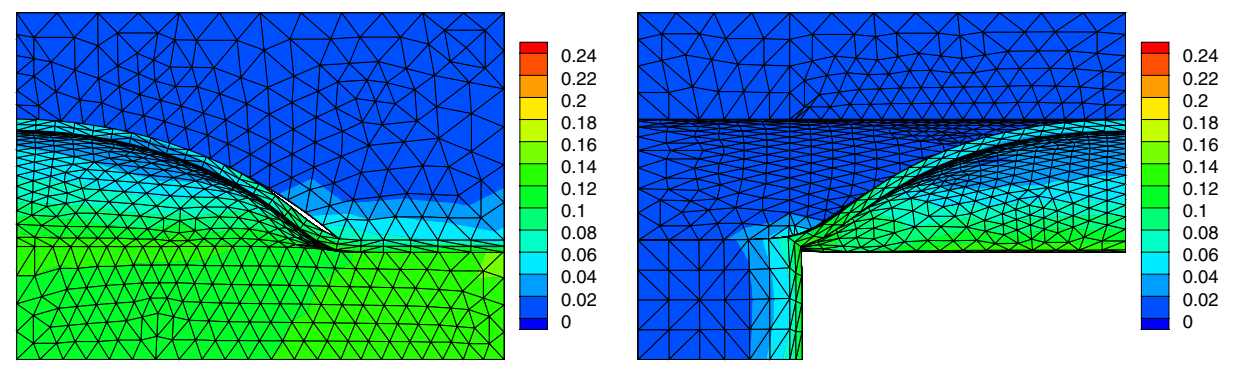

(b)

Figure 8. Contour levels of the Mises stress $\sigma_{M}$ at valve closed: (a) standard microvalve, $8 \mathrm{kPa}$ actuation pressure, lateral views; (b) bistable microvalve, $2 \mathrm{kPa}$ actuation pressure, lateral views. 


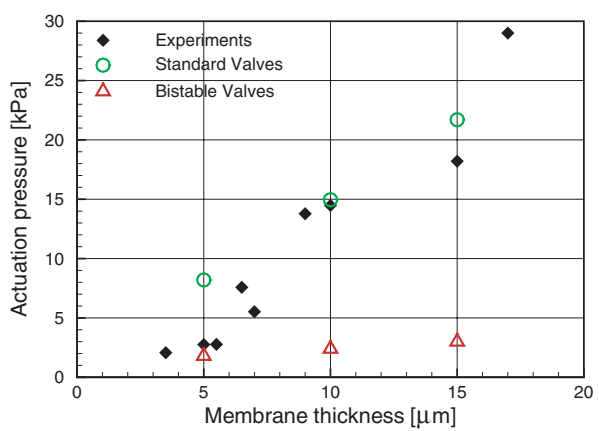

(a)

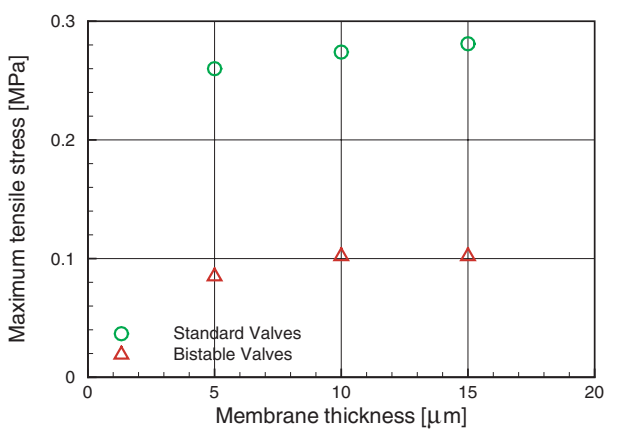

(b)

Figure 9. Comparison between standard (circles) and bistable (triangles) microvalve design. (a) Actuation pressure versus membrane thickness. Black diamonds indicate experimental data on the standard valve design; $(b)$ maximum tensile Cauchy stress in the membrane versus membrane thickness.

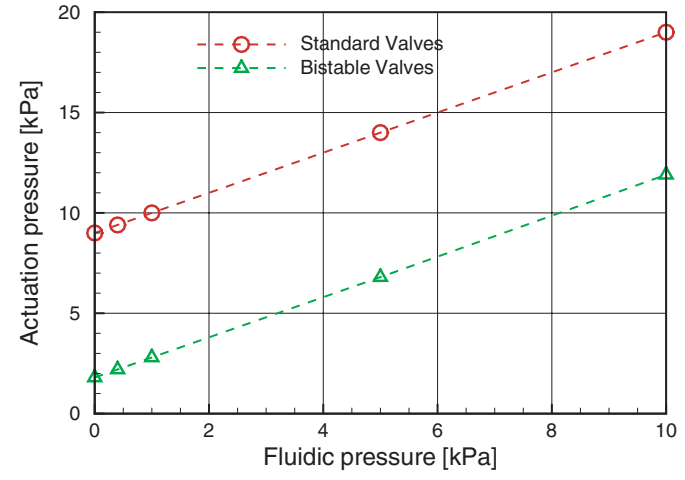

(a)

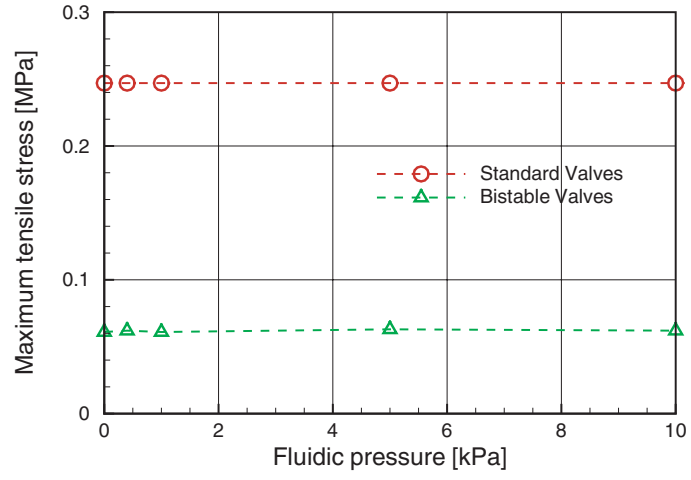

(b)

Figure 10. Effects of the presence of a fluidic pressure: Comparison between standard (circles) and bistable (triangles) microvalve design. (a) Actuation pressure versus fluidic pressure; $(b)$ maximum tensile Cauchy stress in the membrane versus fluidic pressure.

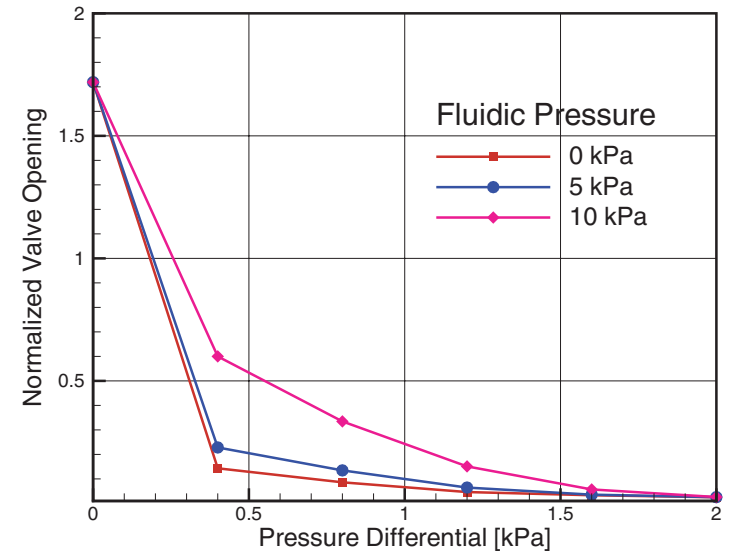

Figure 11. Normalized valve opening of the new design as a function of the difference between actuation and fluidic pressures, and of the value of the fluidic pressure.

by the presence of the fluidic pressure. Contrariwise, the valve opening at intermediate pressure differentials is sensitive to the magnitude of the fluidic pressure. Note that at zero pressure the normalized valve opening values are greater than 1 because the valve buckles in the open configuration.

As an additional design concern, we note that for thick membranes $(\geqslant 10 \mu \mathrm{m})$ the valve closure is not perfect on the lateral side, where the small gap remains. This gap is due to the swelling of the PDMS, which impedes the folding of the
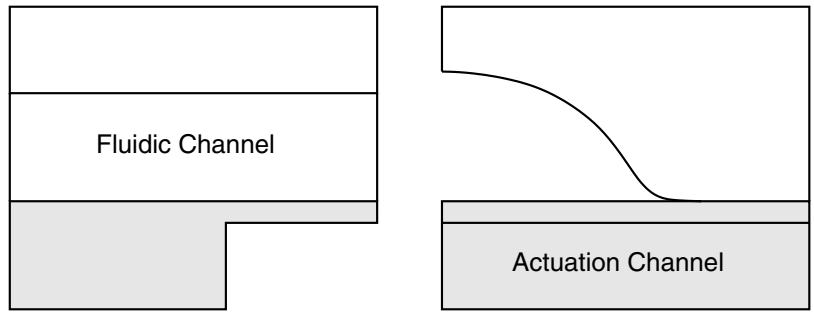

Figure 12. Alternative smooth profile of the fluidic channel, allowing for the complete valve closure.

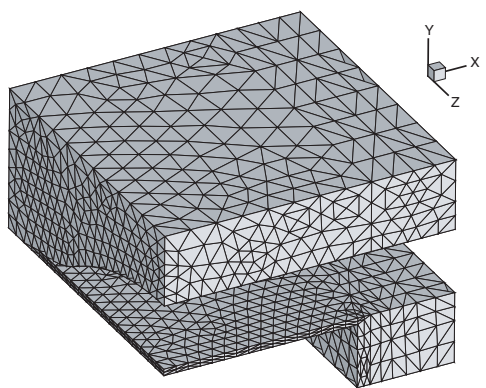

Figure 13. Alternative mesh with a smoothed profile, consisting of 3093 nodes and 1557 ten-node tetrahedra.

membrane at the intersection with the wall. This difficulty can be overcome by smoothing the shape of the fluidic channel (figure 12). 

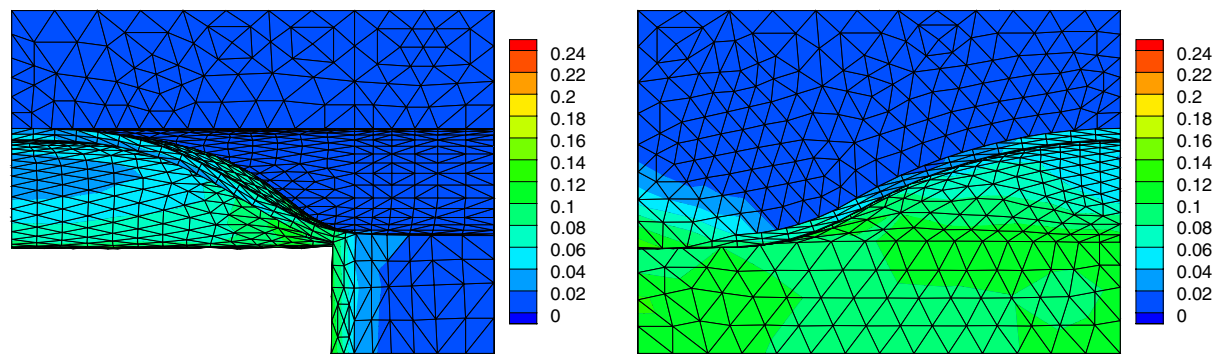

Figure 14. Contour levels of the Mises stress $\sigma_{M}$ at valve closed. Lateral views.

To verify the performance of the smooth profile, we performed an additional analysis with the modified geometry, assuming the same volume change (5\%) and applying the same actuation pressure $(2 \mathrm{kPa})$ as in the case of the circular channel with zero fluidic pressure. The new mesh is shown in figure 13.

As desired, the smooth valve profile has the effect of entirely removing the lateral gap observed in the circular configuration (figure 14).

\section{Concluding remarks}

We have presented an analysis of a new fluidic microvalve design. By exploiting the swelling induced by silicone solvents, the treated membrane can be made to exhibit two stable configurations that can be switched reversibly by the application of modest pressures. One of the buckled configurations partially closes the fluidic channels. The complete closure of the valve can be achieved by applying actuation overpressures that are much reduced in value relative to those required in a standard monostable valve design.

We conclude by noting that chemical swelling of PDMSbased devices has often been regarded as detrimental to microfluidic device performance. Thus, in applications requiring the use of organic solvents excessive swelling may prevent organic solvents from flowing inside the channels, which makes the use of alternative elastomers such as perfluoropolyethers (PEPEs) preferable [8]. We have shown in this paper that controlled chemical swelling can also be used to considerable advantage in the design of microvalves. Specifically, the bistability of the microvalve membrane induced by the chemical swelling results in a much reduced actuation pressure required to switch the valve on and off.

\section{Acknowledgments}

AP acknowledges the support of the Italian MIUR PRIN-2005 programme, protocol no. 2005085973.

\section{References}

[1] Xia Y N and Whitesides G M 1998 Soft lithography Angew. Chem. Int. Ed. 37 550-75

[2] Unger M A, Chou H-P, Thorsen T, Scherer A and Quake S R 2000 Monolithic microfabricated valves and pumps by multiplayer soft lithography Science 228 113-6

[3] Hong J W and Quake S R 2003 Integrated nanoliter systems Nature Biotechnol. 21 1179-83

[4] Studer V, Hang G, Pandolfi A, Ortiz M, Anderson W F and Quake S R 2004 Scaling properties of a low-actuation pressure microfluidic valve J. Appl. Phys. $95393-8$

[5] Quake S R and Scherer A 2000 From micro- to nanofabrication with soft materials Science $2901536-40$

[6] McDonald J C and Whitesides G M 2002 Poly(dimethylsiloxane) as a material for fabrication of microfluidic devices Acc. Chem. Res. 35 491-9

[7] Lee J N, Park C and Whitesides G M 2003 Solvent compatibility of poly(dimethylsiloxane)-based microfluidic devices Anal. Chem. 75 6544-54

[8] Rolland J P, Van Dam R M, Schorzman D A, Quake S R and DeSimone J M 2004 Solvent-resistant photocurable 'Liquid Teflon' for microfluidic device fabrication J. Am. Chem. Soc. Commun. $1262322-3$

[9] Mathison D E, Yates B and Darby M I 1991 Absorption of a silicone fluid in a silicone elastomer J. Mater. Sci. 26 6-10

[10] Underwood P 1983 Dynamic relaxation Computational Methods for Transient Dynamic Analysis ed T Belytschko and T J R Hughes (Amsterdam: Elsevier) pp 245-65

[11] Oakley D R and Knight N F J 1995 Adaptive dynamic relaxation algorithm for non-linear hyperelastic structures: I. Formulation Comput. Methods Appl. Mech. Eng. $12667-89$

[12] Kane C, Repetto E A, Ortiz M and Marsden J E 1999 Finite element analysis of nonsmooth contact Comput. Methods Appl. Mech. Eng. 180 1-26

[13] Pandolfi A, Kane C, Marsden J E and Ortiz M 2002 Time-discretized variational formulation of non-smooth frictional contact Int. J. Numer. Methods Eng. 53 1801-29

[14] Mota A, Klug W S, Ortiz M and Pandolfi A 2003 Finite-element simulation of firearm injury to the human cranium Comput. Mech. 31 115-21

[15] Ogden R W 1997 Non-Linear Elastic Deformations (New York: Dover) 